\title{
Low complexity 2D-DOA estimation algorithm for non-uniform L-shaped array
}

\author{
Yang Donglin ${ }^{1}$, Cheng Qianlin ${ }^{1}$, Lv Weihua $^{1}$, Zheng Wang $^{1}$, Zhang Xiaofei $^{1,2}$ \\ ${ }^{1}$ College of Electronic and Information Engineering, Nanjing University of Aeronautics and \\ Astronautics, Nanjing, 210016, China \\ ${ }^{2}$ National Mobile Communications Research Laboratory, Southeast University,210096,China \\ Email: zhangxiaofei@nuaa.edu.cn
}

Keywords: 2D-direction of arrival estimation; Non-uniform L-shaped array; Multiple signal classification

\begin{abstract}
In this paper, we discussed the problem of two-dimensional (2D) direction of arrival (DOA) estimation for non-uniform L-shaped array, and for this, a low complexity multiple signal classification (MUSIC) algorithm is proposed. The proposed algorithm uses two one-dimensional searches to achieve the joint estimation of 2-dimensional DOA so as to avoid the high computational cost within 2D-MUSIC algorithm. And the angle estimation performance is very close to the 2D-MUSIC algorithm. The proposed algorithm achieves a good balance between the estimation accuracy and complexity.
\end{abstract}

\section{Introduction}

Direction of arrival (DOA) estimation, is a key issue in array signal processing which has been widely applied in military and civil fields, such as communication, radar, sonar and medical etc.[1-4]. Direction of arrival estimation is undoubtedly a key technology in array signal processing. Among the various arrays like the parallel uniform linear array [5], the rectangular array [6], and the circular array [7]. L-shaped array, consisting of two linear subarrays connected orthogonally at one end of each subarray, is a commonly used two-dimensional DOA array. It features simple structure and relatively high estimation accuracy in practice [8]. Meanwhile in recent years, the two-dimensional DOA estimation as a symbol of array signal processing has become a hot spot. Thus, 2D-DOA estimation with L-shaped array has attracted great attention [9-12].

As the most typical two-dimensional DOA algorithm, the two-dimensional MUSIC algorithm needs to search for the peak of the two-dimensional parameter space spectrum. However in practice its application is limited due to computational complexity. In this paper, the proposed algorithm uses two one-dimensional searches to achieve the joint estimation of 2-dimensional DOA so as to avoid the high computational cost within 2D-MUSIC algorithm. And the angle estimation performance is very close to the 2D-MUSIC algorithm.

\section{Data model}

In Fig.1, the non-uniform L-shaped array is consisted of two linear subarrays with $\mathrm{M}$ and $\mathrm{N}$ omnidirectional sensors on the $\mathrm{z}$ - and $\mathrm{x}$ - axes, respectively. We define $\mathrm{Zm} / \mathrm{Xn}(\mathrm{m}=1, \ldots, \mathrm{M}, \mathrm{n}=1, \ldots, \mathrm{N})$ to denote the distance of the $\mathrm{mth} / \mathrm{nth}$ sensor along the $\mathrm{z}$-axis and $\mathrm{x}$-axis from the origin, respectively. We assume there are $\mathrm{K}(\mathrm{K}<\mathrm{M}+\mathrm{N}-1)$ far-field, narrow-band and non-coherent signals impinging on the array with the elevation and azimuth angles $\left(\theta_{k}, \phi_{k}\right), k=(1,2, \ldots, \mathrm{K})$. In this, $\theta_{k}, \phi_{k}$ stands for the elevation and azimuth angles with the range $\left(\theta_{k} \in[0, \pi / 2], \phi_{k} \in[0, \pi]\right)$. Considering the signal sources are distant enough, the arriving far-field signals is regarded as parallel waves. Supposing that exists 


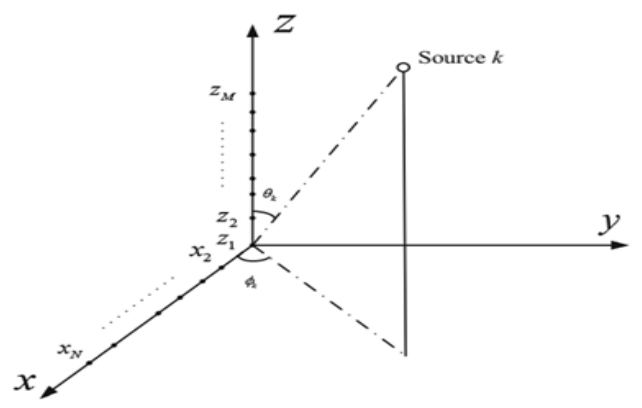

Fig.1 The structure of non-uniform L-shaped array

the additive white Gaussian noise with zero mean and variance $\sigma^{2}$. The received signal of each subarray can be given by:

$$
\begin{aligned}
& \mathbf{Z}=\mathbf{A}_{\mathrm{z}} \mathbf{S}+\mathbf{N}_{\mathrm{z}}, \\
& \mathbf{X}=\mathbf{A}_{\mathrm{x}} \mathbf{S}+\mathbf{N}_{\mathrm{x}},
\end{aligned}
$$

where the steering matrix $\mathbf{S}(t)=\left[s_{1}(t), \ldots, s_{K}(t)\right]^{T} \in \mathbf{C}^{K \times L}, \mathbf{N}_{z} \in \mathbf{C}^{M \times L}, \mathbf{N}_{\mathrm{x}} \in \mathbf{C}^{N \times L}$, L denotes the length of signal. The steering matrices $\mathbf{A}_{z} \in \mathbf{C}^{M \times K}$ and $\mathbf{A}_{x} \in \mathbf{C}^{N \times K}$ are given by: $\mathbf{A}_{z}=\left[\mathbf{a}_{z}\left(\theta_{1}, \phi_{1}\right), \ldots, \mathbf{a}_{z}\left(\theta_{k}, \phi_{k}\right)\right]$, $\mathbf{A}_{\mathbf{x}}=\left[\mathbf{a}_{\mathbf{x}}\left(\theta_{1}, \phi_{1}\right), \ldots, \mathbf{a}_{\mathbf{x}}\left(\theta_{k}, \phi_{k}\right)\right]$, where $\mathbf{a}_{z}\left(\theta_{k}, \phi_{k}\right)=\left[v_{M, k}, \ldots, v_{1, k}\right]^{T}, \mathbf{a}_{\mathbf{x}}\left(\theta_{k}, \phi_{k}\right)=\left[\mu_{1, k}, \ldots, \mu_{N, k}\right]^{T}$, $v_{m, k}=e^{j \alpha_{k} z_{m}}, \mu_{n, k}=e^{j \beta_{k} x_{n}}, \alpha_{k}=2 \pi \cos \theta_{k} / \lambda, \beta_{k}=2 \pi \cos \phi_{k} \sin \theta_{k} / \lambda$.

\section{Traditional 2D-DOA estimation algorithm}

According to the formula (1) and (2), construct matrix for received signal:

$$
\mathbf{Y}=\left[\begin{array}{l}
\mathbf{X} \\
\mathbf{Z}
\end{array}\right]=\mathbf{A}(\theta, \phi) \mathbf{S}+\mathbf{N}_{\mathrm{Y}}=\left[\begin{array}{l}
\mathbf{A}_{\mathrm{x}} \\
\mathbf{A}_{\mathrm{z}}
\end{array}\right] \mathbf{S}+\mathbf{N}_{\mathrm{Y}},
$$

where $\mathbf{N}_{\mathrm{Y}}$ denotes the matrix of received noise. By constructing covariance matrix, which can be estimated by $\hat{\mathbf{R}}$, the noise subspace is obtained, where $\hat{\mathbf{R}}=\frac{1}{\mathrm{~L}} \sum_{1}^{\mathrm{L}} \mathbf{Y} \mathbf{Y}^{\mathrm{H}}$.

Due to the fact that all column vectors of the matrix $\mathbf{A}(\theta, \phi)$ is orthogonal to the noise subspace, spatial spectrum function is given by:

$$
P_{2 D-M U S I C}(\theta, \phi)=\frac{1}{\mathbf{a}(\theta, \phi)^{H} \mathbf{U}_{\mathbf{N}} \mathbf{U}_{\mathbf{N}}{ }^{H} \mathbf{a}(\theta, \phi)},
$$

where $\mathbf{a}(\theta, \phi)=\left[\begin{array}{c}\mathrm{e}^{\mathrm{j} 2 \pi \cos \theta \sin \phi X_{n} / \lambda} \\ \mathrm{e}^{\mathrm{j} 2 \pi \cos \theta Z_{m} / \lambda}\end{array}\right], \mathbf{U}_{\mathrm{N}}=\left[\begin{array}{c}\mathbf{U}_{\mathrm{NX}} \\ \mathbf{U}_{\mathrm{NZ}}\end{array}\right]$ is the noise subspace of the received signal.

With this function, search for the two-dimensional spectrum peak to obtain the DOA,which has the disadvantage of large computational complexity. In next section, a low complexity MUSIC algorithm is proposed to solve the problem.

\section{Low complexity MUSIC algorithm}

According to the introduction of the data model of the non-uniform L-shaped array, the received signal of the subarray on the Z-axis is only related to the elevation angle of the angle pairs. Thus the first step is to construct the covariance matrix of the received signal of the subarray on the Z-axis. The spatial spectrum function is given by:

$$
P_{\mathrm{MUSIC}}(\theta)=\frac{1}{\mathbf{a}(\theta)^{H} \mathbf{U}_{\mathrm{NZ}} \mathbf{U}_{\mathrm{NZ}}{ }^{H} \mathbf{a}(\theta)},
$$


where $\mathbf{a}(\theta)=\mathrm{e}^{\mathrm{j} 2 \pi \cos \theta Z_{m} / \lambda}, \mathbf{U}_{\mathrm{NZ}}$ is the noise subspace of the signal received on the Z-axis.

By spatial spectrum peak search, the initial value $\hat{\theta}_{k}^{\text {ini }}$ of elevation angles is obtained. With the initial estimation of elevation angles $\left\{\hat{\theta}_{k}^{\text {in }}\right\},(k=1, \ldots, K)$, we can furtherly estimate azimuth angle of the $k$ th target, i.e., $\left\{\widehat{\phi}_{k}\right\},(k=1, \ldots, K)$ by keeping the $k$ th elevation angle fixed to $\hat{\theta}_{k}^{i n i}$ and searching the peak of the following $1 \mathrm{D}$ spectrum globally

$$
P_{\text {MUSIC }}(\phi)=\frac{1}{\mathbf{a}\left(\hat{\theta}_{k}^{i n i}, \phi\right)^{H} \mathbf{U}_{\mathrm{N}} \mathbf{U}_{\mathrm{N}}{ }^{H} \mathbf{a}\left(\hat{\theta}_{k}^{i n i}, \phi\right)},
$$

where $\mathbf{a}\left(\hat{\theta}_{k}^{i n i}, \phi\right)=\left[\begin{array}{c}\mathrm{e}^{\mathrm{j} 2 \pi \cos \hat{\theta}_{k}^{\mathrm{in} i} \sin \phi X_{n} / \lambda} \\ \mathrm{e}^{\mathrm{j} 2 \pi \cos \hat{\theta}_{k}^{i n i} Z_{m} / \lambda}\end{array}\right]$.

\section{Complexity Analysis}

The computational complexity of the 2D-MUSIC and the proposed algorithms are analyzed simply as following, where we consider chiefly on the complex multiplication. It is the major computation in the algorithms, and mainly results from the covariance matrix, eigenvalue-decomposition and search of spectral peak.

We define $\mathrm{n}_{1}$ and $\mathrm{n}_{2}$ as the times of the spectrum search of azimuth angle and elevation angle, respectively. The computational complexity of 2D-MUSIC algorithm is $\mathrm{O}\left\{\mathrm{LM}^{2} \mathrm{~N}^{2}+\mathrm{M}^{3} \mathrm{~N}^{3}+\mathrm{n}_{1} \mathrm{n}_{2}(\mathrm{~N}-\mathrm{K})\left(2 \mathrm{M}^{2}-\mathrm{N}-\mathrm{K}\right)\right\}$, while the computational complexity of our proposed algorithm is $\mathrm{O}\left\{\left(\mathrm{LM}^{2} \mathrm{~N}^{2}+\mathrm{M}^{3} \mathrm{~N}^{3}\right)+\left(\mathrm{n}_{1}+\mathrm{n}_{2}\right)(\mathrm{N}-\mathrm{K})\left(2 \mathrm{M}^{2}-\mathrm{N}-\mathrm{K}\right)\right\}$.

Fig. 2 shows the comparison of complexity between the two algorithms. We assume $N=8, K=2$, $\mathrm{L}=300$ and let $\mathrm{M}$ increase from 5 to $20 . \mathrm{n}_{1}$ and $\mathrm{n}_{2}$ are set to be 1000 and 500 . From this we can conclude that our proposed algorithm has much lower computational cost than general 2D-MUSIC algorithm.

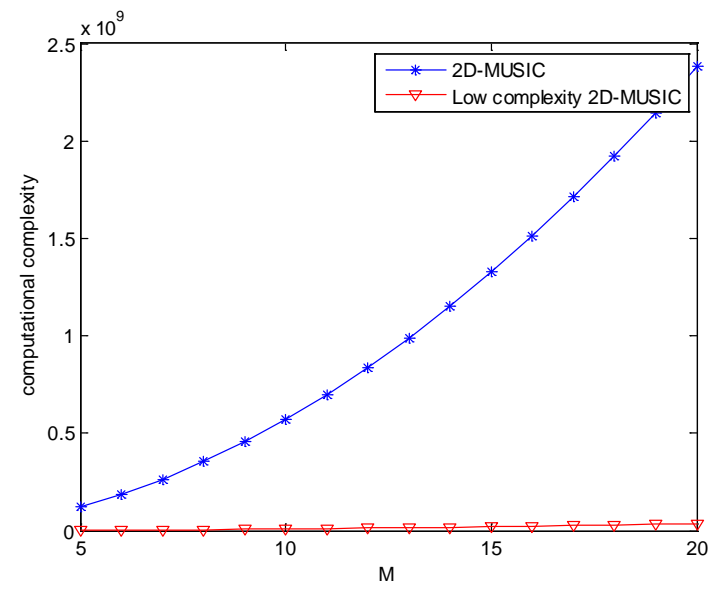

Fig.2 Comparison of complexity between 2D-MUSIC and the proposed algorithm.

\section{Simulation Results}

In this section, Monte Carlo simulations are conducted to assess the DOA estimation performance of the proposed algorithm. Define root mean square error (RMSE) of elevation/azimuth angles as 


$$
R M S E=\frac{1}{\mathrm{~K}} \sum_{1}^{\mathrm{K}} \sqrt{\frac{1}{T} \sum_{n=1}^{T}\left[\left(\theta_{\mathrm{n}}-\theta\right)^{2}+\left(\phi_{\mathrm{n}}-\phi\right)^{2}\right]},
$$

where $\theta_{\mathrm{n}}$ and $\phi_{\mathrm{n}}$ are the estimates of elevation/azimuth angles of the $n$th Monte Carlo trial, respectively. And its trial number $\mathrm{T}$ is set as 1000.Non-uniform linear L-shaped array with $\mathrm{dx}=[0.9$

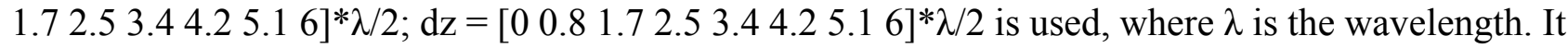
is seen that there are $\mathrm{N}=8$ sensors on the $x$-axis and $\mathrm{M}=8$ sensors on the $z$-axis.We assume there are 2 far-field, narrow-band and non-coherent signal sources locating at $\left(35^{\circ}, 10^{\circ}\right)$ and $\left(15^{\circ}, 40^{\circ}\right)$, respectively, impinging on the array. Note that snapshot $\mathrm{L}=300$.

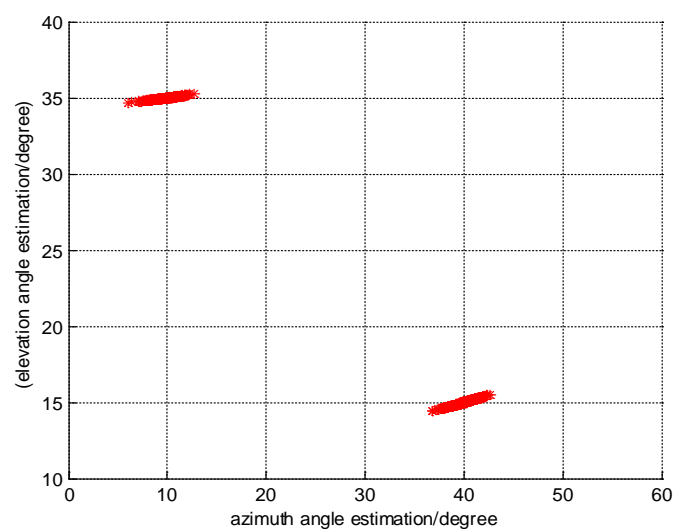

Fig.3 DOA estimation of the proposed algorithm with $\mathrm{SNR}=20 \mathrm{~dB}$

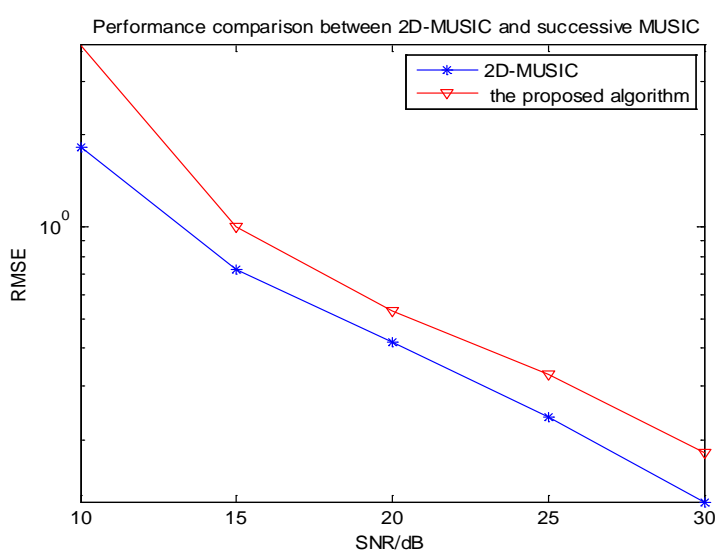

Figure.5 Performance comparison between 2D-MUSIC and the proposed algorithm

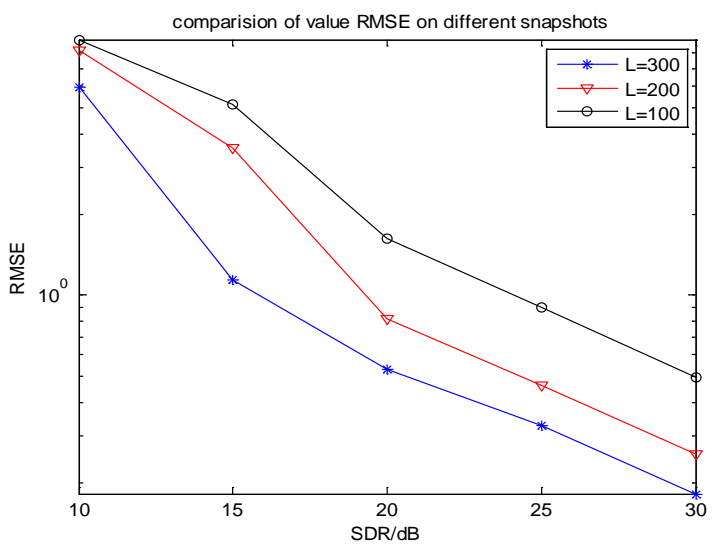

Fig.4 Performance comparison with different snapshots

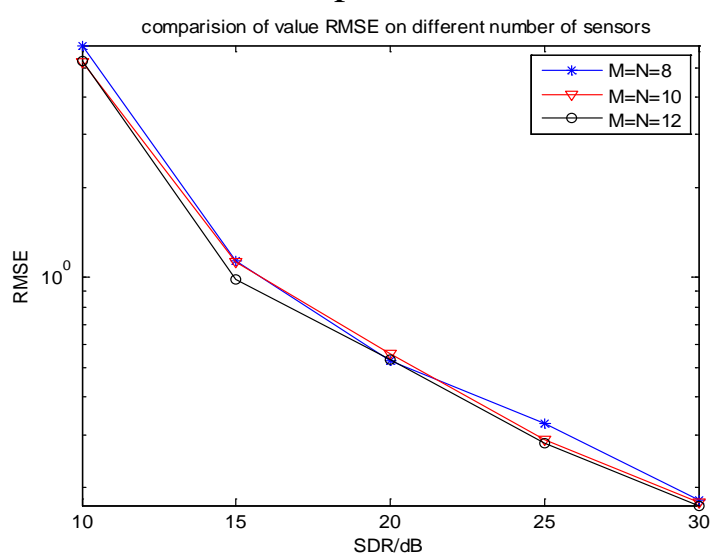

Figure.6 Performance comparison with different number of sensors

Fig.3 demonstrates the estimation results with 100 Monte Carlo runs with SNR=20dB . From this, it can be seen that the angle pairs distribute near the theoretical location of the sources, which verifies the effectiveness of the proposed algorithm..

Fig.4 presents the performance versus snapshot number. Seemly,it is indicated that the angle estimation performance of the proposed algorithm becomes better with snapshot number increasing.

Fig.5 illustrates the performance of the proposed algorithm relative to the 2D-MUSIC. Our proposed algorithm has a very close estimation performance to the 2D-MUSIC algorithm and notably, the proposed algorithm only needs a much lower computational complexity.

Fig.6 depicts the estimation performance with different numbers of sensors. From the illustration, we can conclude that with the increase of the sensors number, the angle estimates become more accurate,but the influence are not significant. 


\section{Conclusion}

A low complexity 2D-MUSIC algorithm has been proposed for two dimensional DOA estimation on the non-uniform L-shaped array. Instead of making 2D spatial spectrum peak search, we obtain the initial elevation angle from one dimensional search from the signal subspace and achieve one-dimensional local searches to complete the joint estimation of the angles. Our proposed method has greatly reduced the computational cost comparing with 2D-MUSIC. Meanwhile, the proposed algorithm has very close estimation performance to the 2D-MUSIC algorithm. Moreover, it has no constraints on the element spacing, and it is suitable for non-uniform linear array.

\section{Acknowledgments}

This work is supported by China NSF Grants (61371169, 61271327), the open research fund of National Mobile Communications Research Laboratory, Southeast University (no.2015D03), Funding of Jiangsu Innovation Program for Graduate Education (KYLX15 0281), Funding for Outstanding Doctoral Dissertation in NUAA (BCXJ15-03), Qing Lan Project, and priority academic pro-gram development of Jiangsu high education institutions.

\section{References}

[1] Zhang X F, Wang F, Chen H W. Theory and application of array signal processing [M] 2nd ed. Beijing: Defense Industry press,2013.

[2] Kim J M, Lee O K, Ye J C. Compressive MUSIC: revisiting the link between compressive sensing and array signal processing[J]. IEEE trans. On information theory, 2012,58(1):278-301.

[3] Naidu P S. Sensor array signal processing[M]. Florida: CRC Press,2010.

[4] Liu Z, Ruan X, He J. Efficient 2-D DOA estimation for coherent sources with a sparse acoustic vector-sensor array[J]. Multidimensional Systems \& Signal Processing, 2013,24(1):105-120.

[5] Yin, Q. Y., Newcomb, R. W., \& Zou, L. H. (1989, May). Estimating 2-D angles of arrival via two parallel linear arrays. In 1989 IEEE International Conference on Acoustics, Speech, and Signal Processing, 2803-2806.

[6] Zoltowski, M. D., Haardt, M., \& Mathews, C. P. (1996). Closed-form 2-D angle estimation with rectangular arrays in element space or beamspace via unitary ESPRIT. IEEE Transactions on Signal Processing, 44(2), 316-328.

[7] Ye, Z., Xiang, L., \& Xu, X. (2007). DOA estimation with circular array via spatial averaging algorithm. IEEE Antennas and Wireless Propagation Letters, 6, 74-76.

[8] Hua, Y., Sarkar, T. K., \& Weiner, D. D. (1991). An L-shaped array for estimating 2-D directions of wave arrival. IEEE transactions on antennas and propagation, 39(2), 143-146.

[9] Wu, Y., Liao, G., \& So, H. C. (2003). A fast algorithm for 2-D direction-of-arrival estimation. Signal processing, 83(8), 1827-1831.

[10] Tayem, N., \& Kwon, H. M. (2005). L-shape 2-dimensional arrival angle estimation with propagator method. IEEE Transactions on Antennas and Propagation, 53(5), 1622-1630.

[11] Liang, J., Zeng, X., Wang, W., \& Chen, H.. L-shaped array-based elevation and azimuth direction finding in the presence of mutual coupling. Signal Processing, 91(5), 1319-1328.

[12] Xiaofei, Z., Jianfeng, L., \& Lingyun, X.. Novel two-dimensional DOA estimation with L-shaped array. EURASIP Journal on Advances in Signal Processing, 2011(1), 1-7. 